\title{
Influence of a positive family history of both type 2 diabetes and PCOS on metabolic and endocrine parameters in a large cohort of PCOS women
}

\author{
Elisabeth Lerchbaum ${ }^{1,2}$, Verena Schwetz ${ }^{1}$, Albrecht Giuliani ${ }^{3}$ and \\ Barbara Obermayer-Pietsch ${ }^{1}$
}

${ }^{1}$ Division of Endocrinology and Metabolism, Department of Internal Medicine, Medical University of Graz, $8036 \mathrm{Graz}$, Austria, ${ }^{2}$ University Women's Hospital, 69120 Heidelberg, Germany and ${ }^{3}$ Department of Obstetrics and Gynecology, Medical University of Graz, $8036 \mathrm{Graz}$, Austria
Correspondence should be addressed to E Lerchbaum Email elisabeth.lerchbaum@ medunigraz.at

\begin{abstract}
Objective: There is evidence suggesting a strong genetic background of polycystic ovary syndrome (PCOS). We aim to study the metabolic and endocrine characteristics of PCOS women with and without a family history (FHx) of type 2 diabetes mellitus (T2DM) and PCOS.

Design: Cross-sectional study.

Methods: We analysed the association of T2DM FHx and PCOS FHx with metabolic and endocrine parameters in 714 PCOS women.

Results: A positive FHx of T2DM and PCOS were prevalent in 36.8 and $21.4 \%$ of PCOS women respectively. We found an independent association of T2DM FHx with central fat accumulation, obesity, prediabetes, metabolic syndrome (MS), insulin resistance, low HDL and elevated blood pressure $(P<0.05$ for all). PCOS FHx was independently associated with prediabetes $(P<0.05)$. We observed an independent association of PCOS FHx with clinical and biochemical hyperandrogenism $(P<0.05$ for all), whereas there was no independent association of T2DM FHx with hyperandrogenism. PCOS women with a positive FHx of both T2DM and PCOS had an adverse metabolic and endocrine profile including a linear increase in risk of obesity, central fat accumulation, MS, prediabetes and low HDL $(P<0.05$ for all).

Conclusions: Our findings suggest that the assessment of FHx might allow risk stratification of PCOS women, which is important considering the high prevalence of PCOS.
\end{abstract}

\section{Introduction}

Polycystic ovary syndrome (PCOS) is the most common endocrine disease in women of reproductive age, affecting up to $20 \%$ of all women when using Rotterdam criteria (1). There are several lines of evidence suggesting a strong genetic background of PCOS, which is also reflected by a familial accumulation of PCOS cases $(2,3,4,5)$. Thus, a positive FHx of PCOS is considered as a risk factor for PCOS development. PCOS women present not only with hyperandrogenism but also affected by an increased metabolic risk which might be
(C) 2014 European Society of Endocrinology Printed in Great Britain related to obesity as well as to genetic and environmental factors (6). There is accumulating evidence suggesting that central obesity is the key factor in the development of metabolic and cardiovascular risk in PCOS women $(7,8,9)$. In this context, several indices such as lipid accumulation product (LAP) $(8,10)$ and visceral adiposity index (VAI) (11) have been shown to be associated with an increased risk of insulin resistance, prediabetes and type 2 diabetes mellitus (T2DM) in PCOS as well as in other cohorts. Further, there is 
evidence showing that a positive family history (FHx) of T2DM, as a reflection of genetic risk, is associated with an increased risk of the development of T2DM in PCOS women $(12,13,14)$. In PCOS women, obesity and T2DM-related genes $(3,15)$ as well as genetic polymorphisms related to hyperandrogenism (16) have been shown to be associated with PCOS phenotype, suggesting an important genetic background. Apart from this, it has not been investigated so far whether a positive FHx of T2DM and PCOS contribute equally to metabolic and endocrine disturbances in PCOS women or whether there are differences regarding the impact of T2DM and PCOS FHx on metabolic and endocrine parameters. Further, most previous studies investigating the association of T2DM FHx with PCOS phenotype included women from the USA who are more obese and have a higher prevalence of metabolic disturbances compared with European cohorts $(12,13,14)$.

The aim of the study was to:

i) study the metabolic and endocrine characteristics of central European PCOS women with and without a FHx of T2DM and PCOS respectively; and

ii) investigate the association of central fat accumulation reflected by LAP and VAI with a positive FHx of T2DM and PCOS respectively.

\section{Subjects and methods}

\section{Subjects}

The study cohort consisted of 714 Caucasian White women with PCOS, aged 16-45 years, and 139 BMImatched control women within the same age range. PCOS women were routinely referred to our outpatient clinic for evaluation of PCOS from 2006 to 2012. PCOS women consulted our outpatient clinic for PCOS-related symptoms such as hirsutism, acne, obesity, infertility or menstrual irregularities or were referred to our outpatient clinic by gynaecologists for further endocrine and metabolic evaluation because of polycystic ovaries. PCOS was diagnosed using the Rotterdam criteria (17). Two out of the following three characteristics are required to confirm the diagnosis: clinical and/or biochemical signs of hyperandrogenism, oligo- and/or anovulation and polycystic ovaries (by ultrasound). Hyperandrogenism was defined by the clinical presence of hirsutism (modified FerrimanGallwey (FG) score $\geq 6$ ), acne or alopecia and/or elevated androgen levels (normal range of total testosterone: $<0.77 \mathrm{ng} / \mathrm{ml}$, free testosterone: $<0.013 \mathrm{ng} / \mathrm{ml}$, and free androgen index (FAI): <5.5). Oligo- and/or anovulation were defined by the presence of oligomenorrhoea or amenorrhoea. Polycystic ovarian morphology was examined by ultrasound (17). Hyperprolactinaemia, Cushing's syndrome, congenital adrenal hyperplasia and androgen-secreting tumours were excluded by specific laboratory analysis (prolactin, cortisol, adrenocorticotropic hormone (ACTH) and $17 \alpha-\mathrm{OH}$ progesterone).

Healthy women, who were routinely referred to our outpatient clinic for thyroid evaluation between 2009 and 2010, were included in the study as a control group. All control women had normal thyroid function, regular menstrual cycles, normal serum androgens and no clinical signs of hyperandrogenism. PCOS and control women did not take any medication known to affect endocrine parameters, carbohydrate metabolism or serum lipid profile for at least 3 months before entering the study.

The study protocol was approved by the ethics committee of the Medical University of Graz and written informed consent was obtained from each patient.

\section{Procedures}

We assessed FHx of PCOS using questionnaires. PCOS women were asked the following questions: have your mother or sisters been diagnosed with PCOS? Do your mother or sisters have clinical (hirsutism or acne) or biochemical hyperandrogenism? Do your mother or sisters have menstrual irregularities or PCO? Similarly, FHx of T2DM was assessed using questionnaires asking whether T2DM is prevalent in brothers, sisters, parents or grandparents of PCOS women and control women respectively.

Standard anthropometric data (height, weight, waist circumference (WC) and hip circumference, blood pressure (BP)) were obtained from each subject. WC was measured in a standing position midway between the lower costal margin and the iliac crest. Hip circumference was measured in a standing position at the maximum circumference over the buttocks. The BMI was calculated as the weight in kilograms divided by the square of height in meters. Hirsutism was quantified with the modified FG score.

Basal blood samples for hormonal (total testosterone, sex-hormone-binding globulin (SHBG), androstenedione, DHEAS, free tri-iodothyronine $\left(\mathrm{fT}_{3}\right)$, free thyroxine $\left(\mathrm{fT}_{4}\right)$, $\mathrm{TSH}, 17 \alpha-\mathrm{OH}$ progesterone, prolactin, cortisol, ACTH), glucose, HbA1c and serum lipids were collected between 0800 and $0900 \mathrm{~h}$ after an overnight fast in the follicular phase in women with regular menses or at random in women with menstrual irregularities. Free testosterone values were calculated from total testosterone, SHBG and albumin according to Vermeulen (18). The FAI was 
calculated as total testosterone $(\mathrm{nmol} / \mathrm{l}) / \mathrm{SHBG}(\mathrm{nmol} / \mathrm{l}) \times$ 100. All participants underwent a fasting $75 \mathrm{~g}$ oral glucose tolerance test (OGTT). Blood samples were drawn after 30, 60 and $120 \mathrm{~min}$ for glucose and insulin determination. The area under the glucose response curve (AUCgluc) and the area under the insulin response curve (AUCins) were calculated according to the trapezoidal method.

Obesity was defined as BMI $\geq 30 \mathrm{~kg} / \mathrm{m}^{2}$. LAP was calculated using the formula $(\mathrm{WC}(\mathrm{cm})-58) \times($ triglycerides (TG) $(\mathrm{mmol} / \mathrm{l}))$. Elevated LAP was defined as $>44.1$ as described previously in a large PCOS cohort as best cut-off for detecting disturbed glucose tolerance (8). VAI was calculated using the formula $(\mathrm{WC}(\mathrm{cm}) /(36.58$ $\left.\left.+\left(1.89 \times \mathrm{BMI}\left(\mathrm{kg} / \mathrm{m}^{2}\right)\right)\right)\right) \times(\mathrm{TG}(\mathrm{mmol} / \mathrm{l}) / 0.81) \times(1.52 / \mathrm{HDL}$ $(\mathrm{mmol} / \mathrm{l}))$. Elevated VAI was defined as VAI $>1.675$ as described previously (11). Insulin resistance was estimated using the homeostatic model assessment-insulin resistance (HOMA-IR) and was assumed for levels $>2.5$. Quantitative insulin sensitivity check index (QUICKI) was used for estimation of insulin sensitivity (11). We further calculated insulin sensitivity index Matsuda (ISI (Matsuda)) as an insulin sensitivity index that reflects a composite estimate of hepatic and muscle insulin sensitivity determined from OGTT data (ISI (Matsuda) = $10000 / \sqrt{ }\left((\right.$ FG $\times$ fasting insulin $) \times$ (glucose $_{\text {OGTTmean }} \times$

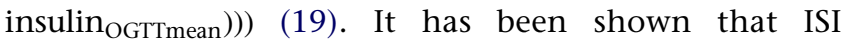
(Matsuda) correlates reasonably well with the estimates of whole-body insulin sensitivity determined by the glucose clamp. The fasting component reflects hepatic insulin sensitivity, whereas the mean of the dynamic data primarily represents skeletal muscle insulin sensitivity. This partitioning concept has recently been validated using glucose clamp studies (20).

Prediabetes and T2DM were defined according to the American Diabetes Association (ADA) (21). Elevated TG levels were defined as TG $>150 \mathrm{mg} / \mathrm{dl}$ and low HDL as HDL $<50 \mathrm{mg} / \mathrm{dl}$. Elevated BP was defined as systolic BP $>130 \mathrm{mmHg}$ and/or diastolic BP $>85 \mathrm{mmHg}(22,23)$. The MS was defined by the National Cholesterol Education Program and the Adult Treatment Panel-III in women presenting at least three of the following criteria: WC $>88 \mathrm{~cm}$, HDL cholesterol $<50 \mathrm{mg} / \mathrm{dl}, \mathrm{TG}$ level $>150 \mathrm{mg} / \mathrm{dl}$, raised blood pressure (systolic $>130 \mathrm{mmHg}$, diastolic $>85 \mathrm{mmHg}$ ), and raised FG $(>110 \mathrm{mg} / \mathrm{dl})$ or prevalent T2DM.

\section{Biochemical analyses}

Total testosterone, insulin, prolactin, TSH, $\mathrm{fT}_{3}, \mathrm{fT}_{4}$ and cortisol (Siemens, Erlangen, Germany) were measured by luminescence immunoassay (intra- and interassay coeffcients of variation (CV values) of $<10 \%)$. SHBG was measured by luminescence immunoassay (Roche) with an intra- and interassay CV of 1.3 and 2.1\% respectively. Albumin was measured by photometric assay (Roche). DHEAS (LDN Labor Diagnostika Nord GmbH, Nordhorn, Germany), androstenedione and $17 \alpha-\mathrm{OH}$ progesterone (DiaMetra, BioVendor, Brno, Czech Republic) were measured by ELISA with intra- and interassay $\mathrm{CV}$ of $<10 \%$.

\section{Statistical analyses}

Data are presented as median with interquartile range unless otherwise stated. The distribution of data was analysed by descriptive statistics and Kolmogorov-Smirnov test. All parameters were found to be non-normally distributed. Kruskal-Wallis test (adjusted for multiple comparisons), Mann-Whitney $U$ test and $\chi^{2}$ test were used for comparisons between groups. We calculated binary logistic regression analyses using all-factors models with various binary metabolic variables as dependent variables and FHx of T2DM (or FHx of PCOS), age and testosterone as independent variables. Moreover, we calculated binary logistic regression analyses using biochemical and clinical hyperandrogenism as dependent variable and age, BMI and T2DM FHX (or PCOS FHx) as independent variables. Age, BMI and total testosterone were log-transformed and rechecked for normal distribution before being entered in logistic regression analyses. The variables followed normal distribution after log-transformation. We further carried out subgroup analyses of obese and non-obese women, repeating the analyses as described earlier.

All statistical procedures were carried out with SPSS version 20 (SPSS, Inc.). $P$ value $<0.05$ was considered statistically significant.

\section{Results}

A positive FHx of T2DM and a positive PCOS FHx were prevalent in 36.8 and $21.4 \%$ of PCOS women respectively. Conversely, $35.1 \%$ of women with normal glucose tolerance had a positive FHx of T2DM, whereas $48.7 \%$ of PCOS women with prediabetes had a positive FHx of T2DM (odds ratio (OR) $1.75(1.08-2.84) ; P=0.023)$ and $44.4 \%$ of PCOS women with T2DM had a positive FHx of T2DM $(P=0.639)$. Baseline characteristics of subjects stratified by FHx of T2DM and PCOS are shown in Tables 1 and 2. Hyperandrogenism, menstrual irregularities and PCO were present in $50.6 \%$ of PCOS women, $32.9 \%$ had hyperandrogenism and menstrual irregularities, $9.8 \%$ had 
Table 1 Characteristics of PCOS women stratified by FHx of T2DM. Analyses were carried out using Mann-Whitney U test or $\chi^{2}$ test.

\begin{tabular}{|c|c|c|c|c|c|c|c|}
\hline & \multirow{2}{*}{\multicolumn{2}{|c|}{ All women $(n=714)$}} & \multicolumn{4}{|c|}{ FHx of T2DM } & \multirow[b]{3}{*}{$P$ value } \\
\hline & & & \multicolumn{2}{|c|}{ Negative $(n=451)$} & \multicolumn{2}{|c|}{ Positive $(n=263)$} & \\
\hline & Median & $\begin{array}{l}\text { Interquartile } \\
\text { range }\end{array}$ & Median & $\begin{array}{l}\text { Interquartile } \\
\text { range }\end{array}$ & Median & $\begin{array}{l}\text { Interquartile } \\
\text { range }\end{array}$ & \\
\hline Age (years) & 27 & $23-32$ & 26 & $23-31$ & 27 & $24-31$ & 0.121 \\
\hline BMI $\left(\mathrm{kg} / \mathrm{m}^{2}\right)$ & 24.2 & $21.3-30.1$ & 23.8 & $20.8-29$ & 25.2 & $21.9-32.1$ & 0.001 \\
\hline WC $(\mathrm{cm})$ & 82 & $72-96$ & 80 & $72-94$ & 86 & $73-100$ & 0.011 \\
\hline WHR & 0.79 & $0.73-0.87$ & 0.79 & $0.73-0.86$ & 0.80 & $0.74-0.88$ & 0.119 \\
\hline Systolic BP (mmHg) & 118 & $110-130$ & 117 & $110-127$ & 120 & $110-133$ & 0.008 \\
\hline Diastolic BP $(\mathrm{mmHg})$ & 80 & $74-89$ & 80 & $73-87$ & 81 & $75-90$ & 0.129 \\
\hline Fasting glucose (mg/dl) & 85 & 79-90 & 84 & 79-90 & 85 & 79-91 & 0.097 \\
\hline Glucose 2-h (mg/dl) & 95 & $81-115$ & 93 & $78-110$ & 99 & $85-123$ & 0.001 \\
\hline AUCgluc & 172.3 & 151.3-198.8 & 170.0 & 150.3-191.3 & 178.6 & $152.9-210.1$ & 0.010 \\
\hline $\mathrm{HbA1c}(\%)$ & 5.2 & $5.0-5.3$ & 5.2 & $5.0-5.3$ & 5.1 & $5.0-5.3$ & 0.137 \\
\hline Fasting insulin $(\mu \mathrm{U} / \mathrm{ml})$ & 6.2 & $3.8-9.8$ & 6.0 & $3.7-9.5$ & 6.4 & $4.0-10.8$ & 0.119 \\
\hline Insulin 2-h $(\mu \mathrm{U} / \mathrm{ml})$ & 33.4 & $20.0-58.0$ & 31.3 & $18.5-55.5$ & 37.4 & $21.7-61.8$ & 0.009 \\
\hline AUCins & 61.6 & $39.4-96.7$ & 57.8 & $36.8-96.7$ & 65.6 & 44.4-97.9 & 0.106 \\
\hline HOMA-IR & 1.27 & $0.80-2.17$ & 1.26 & $0.77-2.04$ & 1.30 & $0.82-2.73$ & 0.061 \\
\hline QUICKI & 0.37 & $0.34-0.40$ & 0.37 & $0.34-0.40$ & 0.37 & $0.33-0.40$ & 0.119 \\
\hline ISI (Matsuda) & 7.34 & $4.52-11.34$ & 7.83 & $4.90-11.74$ & 6.76 & $4.13-10.62$ & 0.020 \\
\hline LAP & 19.7 & $10.3-41.3$ & 18.4 & $9.8-35.5$ & 21.9 & $10.8-50.4$ & 0.022 \\
\hline VAl & 0.89 & $0.62-1.53$ & 0.86 & $0.58-1.47$ & 0.98 & $0.65-1.86$ & 0.015 \\
\hline TC (mg/dl) & 178 & 156-199 & 177 & 155-199 & 178 & 159-199 & 0.563 \\
\hline TG (mg/dl) & 75 & 54-103 & 75 & 54-103 & 75 & $55-103$ & 0.345 \\
\hline HDL (mg/dl) & 64 & $53-76$ & 66 & $54-78$ & 61 & $49-74$ & 0.001 \\
\hline LDL (mg/dl) & 100 & $83-118$ & 98 & $82-117$ & 103 & $86-120$ & 0.046 \\
\hline Testosterone (ng/ml) & 0.60 & $0.46-0.79$ & 0.60 & $0.45-0.80$ & 0.60 & $0.46-0.78$ & 0.739 \\
\hline SHBG (nmol/l) & 45.4 & $29.8-63.0$ & 47.1 & $31.8-66.3$ & 41.4 & $27.2-58.8$ & 0.004 \\
\hline FAI & 4.96 & $3.16-7.72$ & 4.80 & $2.96-7.39$ & 5.21 & $3.47-7.96$ & 0.069 \\
\hline Free testosterone (ng/ml) & 0.009 & $0.007-0.0013$ & 0.009 & $0.006-0.0013$ & 0.009 & $0.007-0.014$ & 0.287 \\
\hline FG score & 6 & $2-11$ & 6 & $2-11$ & 6 & $2-12$ & 0.800 \\
\hline Positive FHx of PCOS (\%) & 21.4 & & 16.1 & & 30.6 & & $<0.001$ \\
\hline Obesity (\%) & 25.3 & & 22.0 & & 30.9 & & 0.009 \\
\hline LAP elevated $(\%)$ & 23.5 & & 19.9 & & 28.9 & & 0.012 \\
\hline VAl elevated (\%) & 23.0 & & 20.1 & & 27.4 & & 0.037 \\
\hline Prediabetes $(\%)$ & 12.8 & & 10.4 & & 16.9 & & 0.022 \\
\hline T2DM (\%) & 1.5 & & 1.3 & & 1.8 & & 0.638 \\
\hline MS (\%) & 11.5 & & 8.6 & & 16.4 & & 0.006 \\
\hline TG elevated $(\%)$ & 10.4 & & 9.1 & & 12.6 & & 0.167 \\
\hline HDL low (\%) & 18.8 & & 14.8 & & 25.8 & & 0.001 \\
\hline BP elevated (\%) & 37.4 & & 34.0 & & 42.6 & & 0.035 \\
\hline $\begin{array}{l}\text { Menstrual } \\
\quad \text { irregularities (\%) }\end{array}$ & 85.5 & & 85.6 & & 85.3 & & 0.393 \\
\hline PCO (\%) & 56.8 & & 55.9 & & 58.2 & & 0.582 \\
\hline $\begin{array}{l}\text { Clinical hyperandro- } \\
\text { genism (\%) }\end{array}$ & 72.7 & & 73.5 & & 71.5 & & 0.609 \\
\hline $\begin{array}{l}\text { Biochemical hyperandro- } \\
\text { genism (\%) }\end{array}$ & 51.0 & & 52.8 & & 47.9 & & 0.252 \\
\hline
\end{tabular}

PCOS, polycystic ovary syndrome; FHx, family history; T2DM, type 2 diabetes mellitus; WC, waist circumference; WHR, waist-to-hip ratio; BP, blood pressure; AUC, area under the curve; HOMA, homeostatic model assessment; QUICKI, quantitative insulin sensitivity check index; ISI, insulin sensitivity index; LAP, lipid accumulation product; VAI, visceral adiposity index; SHBG, sex hormone-binding globulin; FAl, free androgen index; FG, Ferriman-Gallwey; TC, total cholesterol; TG, triglycerides; MS, metabolic syndrome; PCO, polycystic ovaries.

menstrual irregularities and PCO and 6.8\% had hyperandrogenism and PCO.

Binary logistic regression analyses investigating the association of various metabolic parameters with FHx of T2DM and PCOS are presented in Table 3. We found an independent association of T2DM FHx with elevated LAP, obesity, prediabetes, MS, insulin resistance, low HDL and elevated BP. Further, PCOS FHx was independently associated with prediabetes.

Binary logistic regression analyses investigating the association of hyperandrogenism with FHx of T2DM and PCOS are shown in Table 4. We observed an independent 
Table 2 Characteristics of PCOS women stratified by FHx of PCOS. Analyses were carried out using Mann-Whitney $U$ test or $\chi^{2}$ test.

\begin{tabular}{|c|c|c|c|c|c|}
\hline & \multicolumn{4}{|c|}{ FHx of PCOS } & \multirow[b]{3}{*}{$P$ value } \\
\hline & \multicolumn{2}{|c|}{ Negative $(n=500)$} & \multicolumn{2}{|c|}{ Positive $(n=136)$} & \\
\hline & Median & Interquartile range & Median & Interquartile range & \\
\hline Age (years) & 27 & $23-31$ & 27 & $23-31$ & 0.587 \\
\hline BMI $\left(\mathrm{kg} / \mathrm{m}^{2}\right)$ & 23.9 & $21.1-29.3$ & 25.3 & $22.1-31.2$ & 0.025 \\
\hline$W C(\mathrm{~cm})$ & 81 & 72-95 & 85 & $75-100$ & 0.038 \\
\hline WHR & 0.79 & $0.73-0.86$ & 0.80 & $0.74-0.88$ & 0.166 \\
\hline Systolic BP (mmHg) & 117 & $110-130$ & 116 & $110-132$ & 0.443 \\
\hline Diastolic BP (mmHg) & 80 & $72-88$ & 80 & $74-86$ & 0.790 \\
\hline Fasting glucose (mg/dl) & 84 & 79-90 & 87 & $82-93$ & 0.001 \\
\hline Glucose 2-h (mg/dl) & 95 & $81-114$ & 102 & $85-124$ & 0.021 \\
\hline AUCgluc & 170.8 & $150.8-192.0$ & 187.8 & $154.8-209.8$ & 0.013 \\
\hline HbA1c (\%) & 5.1 & $5.0-5.3$ & 5.2 & $4.9-5.3$ & 0.560 \\
\hline Fasting insulin $(\mu \mathrm{U} / \mathrm{ml})$ & 6.0 & $3.8-9.6$ & 6.9 & $4.5-11.5$ & 0.057 \\
\hline Insulin 2-h $(\mu \mathrm{U} / \mathrm{ml})$ & 31.3 & $18.5-55.5$ & 37.4 & $21.7-61.8$ & 0.481 \\
\hline AUCins & 61.1 & $39.1-92.3$ & 70.9 & $42.7-108.2$ & 0.141 \\
\hline HOMA-IR & 1.26 & $0.77-2.00$ & 1.46 & $0.97-2.64$ & 0.020 \\
\hline QUICKI & 0.37 & $0.34-0.40$ & 0.36 & $0.33-0.39$ & 0.027 \\
\hline ISI (Matsuda) & 7.28 & $4.65-11.46$ & 6.56 & $3.70-10.14$ & 0.072 \\
\hline LAP & 19.1 & $10.0-38.3$ & 23.3 & $11.4-53.0$ & 0.053 \\
\hline VAI & 0.89 & $0.63-1.48$ & 0.99 & $0.63-1.77$ & 0.272 \\
\hline $\mathrm{TC}(\mathrm{mg} / \mathrm{dl})$ & 178 & 158-198 & 179 & 154-206 & 0.645 \\
\hline TG (mg/dl) & 75 & $55-101$ & 78 & $56-115$ & 0.303 \\
\hline $\mathrm{HDL}(\mathrm{mg} / \mathrm{dl})$ & 65 & $53-77$ & 64 & $53-77$ & 0.707 \\
\hline LDL (mg/dl) & 99 & $82-116$ & 102 & $84-121$ & 0.379 \\
\hline Testosterone (ng/ml) & 0.62 & $0.49-0.80$ & 0.63 & $0.48-0.81$ & 0.555 \\
\hline SHBG (nmol/l) & 46.4 & $31.0-63.5$ & 39.9 & $25.4-55.1$ & 0.001 \\
\hline FAI & 4.79 & $2.96-7.39$ & 5.68 & $3.70-9.43$ & 0.004 \\
\hline Free testosterone $(\mathrm{ng} / \mathrm{ml})$ & 0.009 & $0.006-0.0013$ & 0.011 & $0.008-0.015$ & 0.012 \\
\hline FG score & 6 & $2-11$ & 9 & 4-15 & $<0.001$ \\
\hline Positive FHx of T2DM (\%) & 32.2 & & 52.2 & & $<0.001$ \\
\hline Obesity (\%) & 22.9 & & 28.9 & & 0.147 \\
\hline LAP elevated $(\%)$ & 21.2 & & 29.7 & & 0.049 \\
\hline VAl elevated (\%) & 21.6 & & 27.3 & & 0.182 \\
\hline Prediabetes (\%) & 11.4 & & 21.6 & & 0.005 \\
\hline T2DM (\%) & 1.2 & & 2.5 & & 0.301 \\
\hline MS (\%) & 10.8 & & 14.1 & & 0.307 \\
\hline TG elevated (\%) & 9.6 & & 13.4 & & 0.194 \\
\hline HDL low (\%) & 18.2 & & 20.9 & & 0.486 \\
\hline BP elevated $(\%)$ & 34.5 & & 36.4 & & 0.703 \\
\hline Menstrual irregularities (\%) & 85.1 & & 86.8 & & 0.631 \\
\hline PCO $(\%)$ & 57.0 & & 55.9 & & 0.816 \\
\hline Clinical hyperandrogenism (\%) & 69.9 & & 81.7 & & 0.008 \\
\hline Biochemical hyperandrogenism (\%) & 50.6 & & 52.5 & & 0.711 \\
\hline
\end{tabular}

PCOS, polycystic ovary syndrome; FHx, family history; WC, waist circumference; WHR, waist-to-hip ratio; BP, blood pressure; AUC, area under the curve; HOMA, homeostatic model assessment; QUICKI, quantitative insulin sensitivity check index; ISI, insulin sensitivity index; LAP, lipid accumulation product; VAI, visceral adiposity index; SHBG, sex hormone-binding globulin; FAl, free androgen index; FG, Ferriman-Gallwey; TC, total cholesterol; TG, triglycerides; T2DM, type 2 diabetes mellitus; MS, metabolic syndrome; PCO, polycystic ovaries.

association of PCOS FHx with clinical hyperandrogenism, hirsutism, elevated FAI levels and free testosterone levels, whereas there was no independent association of T2DM FHx with clinical or biochemical hyperandrogenism.

We carried out further analyses stratifying PCOS women into three groups: no positive FHx (53.3\%), positive FHx of T2DM or PCOS (35.3\%9) and positive FHx of T2DM and PCOS (11.2\%) (Table 5). PCOS women with a positive FHx of both T2DM and PCOS had an adverse metabolic and endocrine profile. Moreover, we found a linear increase in risk of obesity, central fat accumulation, MS, prediabetes and low HDL. Binary logistic regression analyses using various metabolic disturbances as dependent variables and $\mathrm{FHx}$ (three groups: no positive FHx (reference); positive FHx of T2DM or PCOS; positive FHx of T2DM and PCOS), age and testosterone as independent variables are shown in Fig. 1. 
Table 3 Multivariate adjusted binary logistic regression analyses for various metabolic risk factors using FHx of T2DM and PCOS as dependent variables. Analyses were carried out by binary logistic regression analyses using all-factors models with metabolic risk factors as binary dependent variables and positive $\mathrm{FHx}$ of T2DM or PCOS as explanatory variables (a negative $\mathrm{FHx}$ was defined as reference). Analyses were adjusted for age and testosterone.

\begin{tabular}{|c|c|c|c|c|}
\hline & \multicolumn{2}{|c|}{ Positive FHx of T2DM ${ }^{a}$} & \multicolumn{2}{|c|}{ Positive FHx of PCOS $^{b}$} \\
\hline & OR $(95 \% \mathrm{Cl})$ & $P$ value & OR $(95 \% \mathrm{Cl})$ & $P$ value \\
\hline LAP elevated & $1.58(1.07-2.34)$ & 0.020 & $1.46(0.92-2.31)$ & 0.107 \\
\hline VAl elevated & $1.47(1.00-2.17)$ & 0.052 & $1.32(0.83-2.09)$ & 0.240 \\
\hline Obesity & $1.57(1.11-2.23)$ & 0.012 & $1.30(0.84-2.00)$ & 0.242 \\
\hline Prediabetes & $1.71(1.05-2.78)$ & 0.031 & $2.08(1.21-3.56)$ & 0.008 \\
\hline T2DM & $1.31(0.35-4.94)$ & 0.695 & $1.90(0.44-8.18)$ & 0.389 \\
\hline MS & $2.07(1.20-3.57)$ & 0.009 & $1.21(0.66-2.21)$ & 0.543 \\
\hline Insulin resistance & $1.69(1.13-2.53)$ & 0.010 & $1.47(0.93-2.31)$ & 0.101 \\
\hline TG elevated & $1.46(0.86-2.47)$ & 0.158 & $1.39(0.77-2.49)$ & 0.277 \\
\hline HDL low & $2.01(1.34-3.02)$ & 0.001 & $1.20(0.75-1.93)$ & 0.453 \\
\hline Elevated BP & $1.45(1.03-2.04)$ & 0.034 & $0.89(0.58-1.38)$ & 0.603 \\
\hline
\end{tabular}

FHx, family history; T2DM, type 2 diabetes mellitus; PCOS, polycystic ovary syndrome; LAP, lipid accumulation product; VAI, visceral adiposity index; MS, metabolic syndrome; TG, triglycerides; BP, blood pressure.

aData available in 714 women.

${ }^{\mathrm{b}}$ Data available in 636 women.

\section{Non-obese women}

We carried out subgroup analyses of non-obese (BMI $<30 \mathrm{~kg} / \mathrm{m}^{2}$ ) and obese (BMI $\geq 30 \mathrm{~kg} / \mathrm{m}^{2}$ ) PCOS women and metabolic characteristics are shown in Table 6. We found a significantly higher prevalence of PCOS FHx $(P=0.016)$ and decreased HDL in non-obese PCOS women with a positive T2DM FHx, whereas the remaining associations were attenuated (data not shown). Non-obese PCOS women with a positive PCOS FHx had a higher prevalence of T2DM FHx $(P=0.016)$, higher FG $(P=0.004)$ and FG scores $(P=0.027)$ and lower SHBG levels $(P=0.018)$. We observed a trend towards a higher prediabetes prevalence $(P=0.062)$, AUCgluc $(P=0.052)$ and FAI levels $(P=0.071)$ in non-obese PCOS women with a positive PCOS FHx. Further, non-obese PCOS women with a positive FHx of T2DM and PCOS were significantly younger $(P=0.027)$, had higher BMI $(P=0.025)$ and FG $(P=0.030)$ levels, a higher prevalence of prediabetes $(P=0.025)$ and lower SHBG levels $(P=0.007)$ and showed a trend towards higher AUCgluc levels $(P=0.053)$.

Binary logistic regression analyses using metabolic and endocrine parameters as dependent variables were attenuated (except decreased HDL (OR for T2DM FHx: $2.12,1.15-3.91 ; P=0.015)$ and hirsutism (OR for PCOS FHx: $1.59,1.00-2.52 ; P=0.050)$ ).

\section{Obese women}

In obese PCOS women, a positive T2DM FHX was significantly associated with a higher prevalence of PCOS FHx $(P<0.001)$, prediabetes $(P=0.040)$, lower SHBG $(P=0.034)$ and HDL $(P=0.030)$ levels, and showed a trend towards higher FG $(P=0.060)$, 2-h glucose $(P=0.058)$ and testosterone levels $(P=0.073)$. A positive PCOS FHx was significantly associated with a higher prevalence of T2DM FHx $(P<0.001)$ and clinical hyperandrogenism $(P=0.020)$, higher levels of 2 -h glucose (0.007), FAI $(P=0.001)$ and FG score $(P<0.0019$ and lower SHBG levels $(0.001))$. Further, FT levels tended to be higher in PCOS women with a positive PCOS FHx $(P=0.084)$. Moreover, a positive $\mathrm{FHx}$ of T2DM and PCOS was significantly associated with higher FG $(P=0.024)$ and FAI $(P=0.014)$ levels and a higher prevalence of prediabetes $(P=0.047)$ and lower SHBG levels $(P=0.002)$. Further, we observed a trend towards higher TG levels $(P=0.062)$ and elevated VAI $(P=0.074)$.

Table 4 Multivariate adjusted binary logistic regression analyses for hyperandrogenism using FHx of T2DM and PCOS as dependent variables. Analyses were carried out by binary logistic regression analyses using all-factors models with hyperandrogenism as binary dependent variable and positive $\mathrm{FHx}$ of T2DM or PCOS as explanatory variables (a negative $\mathrm{FHx}$ was defined as reference). Clinical hyperandrogenism was defined as the presence of hirsutism and/or acne and/or alopecia. Biochemical hyperandrogenism was defined as elevated testosterone and/or free testosterone and/or FAI. Analyses were adjusted for age and BMI.

\begin{tabular}{|c|c|c|c|c|}
\hline & \multicolumn{2}{|c|}{ Positive FHx of T2DM ${ }^{a}$} & \multicolumn{2}{|c|}{ Positive FHx of PCOS $^{b}$} \\
\hline & OR $(95 \% \mathrm{Cl})$ & $P$ value & OR $(95 \% \mathrm{Cl})$ & $P$ value \\
\hline $\begin{array}{l}\text { Clinical hyper- } \\
\text { androgenism }\end{array}$ & $0.84(0.57-1.24)$ & 0.378 & $1.85(1.13-3.03)$ & 0.015 \\
\hline Hirsutism & $0.81(0.57-1.15)$ & 0.235 & $1.80(1.19-2.73)$ & 0.006 \\
\hline $\begin{array}{l}\text { Biochemical } \\
\text { hyper- } \\
\text { androgenism }\end{array}$ & $0.77(0.54-1.09)$ & 0.143 & $1.02(0.66-1.57)$ & 0.935 \\
\hline $\begin{array}{l}\text { Testosterone } \\
\text { high }\end{array}$ & $0.85(0.58-1.24)$ & 0.396 & $1.01(0.65-1.56)$ & 0.973 \\
\hline $\begin{array}{l}\text { Free testo- } \\
\text { sterone high }\end{array}$ & $0.90(0.56-145)$ & 0.671 & $1.68(1.01-2.80)$ & 0.044 \\
\hline FAl high & $0.90(0.62-1.31)$ & 0.592 & $1.64(1.06-2.53)$ & 0.025 \\
\hline
\end{tabular}

FHx, family history; T2DM, type 2 diabetes mellitus; PCOS, polycystic ovary syndrome; FAl, free androgen index.

a Data available in 714 women.

${ }^{b}$ Data available in 636 women. 
Table 5 Characteristics of PCOS women stratified by FHx of T2DM and PCOS respectively, comparing PCOS women without a positive FHx with women with a positive FHx of T2DM or PCOS and to women with a positive FHx of T2DM and PCOS.

\begin{tabular}{|c|c|c|c|c|c|c|c|}
\hline & \multicolumn{2}{|c|}{ Negative FHx $(n=339)$} & \multicolumn{2}{|c|}{$\begin{array}{l}\text { Positive FHx of T2DM or PCOS } \\
\qquad(n=226)\end{array}$} & \multicolumn{2}{|c|}{$\begin{array}{l}\text { Positive FHx of T2DM and } \\
\text { PCOS }(n=71)\end{array}$} & \multirow[b]{2}{*}{$P$ value } \\
\hline & Median & Interquartile range & Median & Interquartile range & Median & Interquartile range & \\
\hline Age (years) & 26 & $22-31$ & 27 & $24-31$ & 27 & $23-31$ & 0.196 \\
\hline BMI $\left(\mathrm{kg} / \mathrm{m}^{2}\right)$ & 23.9 & $20.9-29.1$ & 24.1 & $21.3-29.0$ & $27.6^{\dagger, \neq}$ & $23.1-33.6$ & $<0.001$ \\
\hline$W C(\mathrm{~cm})$ & 80 & $72-93$ & 82 & $72-95$ & $88^{\dagger, \ddagger}$ & $78-105$ & 0.007 \\
\hline WHR & 0.78 & $0.73-0.86$ & 0.79 & $0.74-0.87$ & 0.81 & $0.75-0.89$ & 0.245 \\
\hline Systolic BP (mmHg) & 116 & $109-128$ & 119 & $110-130$ & 120 & $111-138$ & 0.108 \\
\hline Diastolic BP (mmHg) & 80 & $72-87$ & 80 & $73-90$ & 80 & $75-88$ & 0.753 \\
\hline Fasting glucose $(\mathrm{mg} / \mathrm{dl})$ & 84 & $78-90$ & $86^{*}$ & $79-91$ & $87^{\dagger}$ & $82-93$ & 0.004 \\
\hline Glucose 2-h (mg/dl) & 94 & $81-111$ & 97 & $82-123$ & $107^{\dagger, \neq}$ & $90-126$ & 0.003 \\
\hline AUCgluc & 170.0 & $150.3-189.0$ & 172.0 & $151.8-204.0$ & $189.5^{\dagger}$ & $164.5-212.0$ & 0.004 \\
\hline $\operatorname{HbA1c}(\%)$ & 5.1 & $5.0-5.3$ & 5.1 & $5.0-5.3$ & 5.1 & $4.9-5.4$ & 0.706 \\
\hline Fasting insulin $(\mu \mathrm{U} / \mathrm{ml})$ & 6.0 & $3.7-9.5$ & 6.3 & $3.8-10.0$ & $7.4^{\dagger, \neq}$ & $5.3-13.4$ & 0.035 \\
\hline Insulin 2-h $(\mu \mathrm{U} / \mathrm{ml})$ & 32.4 & $20.0-56.0$ & 34.2 & $20.0-57.0$ & 44.1 & $23.4-72.5$ & 0.099 \\
\hline AUCins & 57.4 & $37.4-92.4$ & 64.1 & 39.3-91.4 & 71.8 & $47.1-118.4$ & 0.117 \\
\hline HOMA-IR & 1.27 & $0.76-2.04$ & 1.26 & $0.80-2.28$ & $1.68^{\dagger, \neq}$ & $1.11-2.95$ & 0.009 \\
\hline QUICKI & 0.37 & $0.34-0.40$ & 0.37 & $0.34-0.40$ & $0.35^{\ddagger}$ & $0.33-0.38$ & 0.017 \\
\hline ISI (Matsuda) & 7.72 & $4.88-11.95$ & 6.90 & $4.27-10.77$ & $5.85^{\dagger}$ & $3.09-9.48$ & 0.025 \\
\hline LAP & 18.7 & $10.0-35.7$ & 19.3 & $10.3-39.7$ & $26.5^{\dagger, \neq}$ & $13.2-63.0$ & 0.017 \\
\hline VAl & 0.88 & $0.62-1.49$ & 0.88 & $0.63-1.45$ & $1.10^{\ddagger}$ & $0.75-2.32$ & 0.067 \\
\hline $\mathrm{TC}(\mathrm{mg} / \mathrm{dl})$ & 178 & $156-199$ & 179 & 158-202 & 176 & $156-202$ & 0.840 \\
\hline TG (mg/dl) & 75 & $56-105$ & 75 & $54-98$ & $85^{\ddagger}$ & $62-126$ & 0.083 \\
\hline $\mathrm{HDL}(\mathrm{mg} / \mathrm{dl})$ & 67 & $53-78$ & 63 & $54-76$ & $59^{\dagger}$ & $46-74$ & 0.043 \\
\hline LDL (mg/dl) & 98 & $80-117$ & 101 & $85-117$ & 97 & $84-120$ & 0.212 \\
\hline Testosterone (ng/ml) & 0.62 & $0.49-0.80$ & 0.62 & $0.47-0.80$ & 0.61 & $0.47-0.81$ & 0.969 \\
\hline SHBG (nmol/l) & 47.6 & $31.1-66.3$ & 44.3 & $29.2-62.0$ & $33.0^{\dagger, \ddagger}$ & $24.4-48.5$ & $<0.001$ \\
\hline FAl & 4.67 & $2.84-7.29$ & 4.98 & $3.36-7.75$ & $5.78^{\dagger, \ddagger}$ & $4.21-9.76$ & 0.006 \\
\hline Free testosterone $(\mathrm{ng} / \mathrm{ml})$ & 0.009 & $0.006-0.012$ & 0.010 & $0.007-0.014$ & 0.010 & $0.008-0.015$ & 0.072 \\
\hline FG score & 6 & $2-11$ & 6 & $3-11$ & $8^{\dagger}$ & $4-13$ & 0.040 \\
\hline Obesity (\%) & 21.6 & & 23.5 & & 38.6 & & 0.010 \\
\hline LAP elevated $(\%)$ & 20.4 & & 22.9 & & 35.9 & & 0.035 \\
\hline VAI elevated (\%) & 21.7 & & 20.5 & & 35.3 & & 0.035 \\
\hline Prediabetes (\%) & 10.4 & & 14.4 & & 26.2 & & 0.005 \\
\hline T2DM (\%) & 1.4 & & 1.0 & & 3.1 & & 0.498 \\
\hline MS (\%) & 8.3 & & 14.0 & & 17.6 & & 0.041 \\
\hline TG elevated (\%) & 9.0 & & 10.4 & & 16.9 & & 0.143 \\
\hline HDL low (\%) & 15.8 & & 19.5 & & 31.0 & & 0.011 \\
\hline BP elevated (\%) & 33.7 & & 37.2 & & 41.3 & & 0.479 \\
\hline Menstrual irregularities (\%) & 85.4 & & 85.0 & & 87.3 & & 0.885 \\
\hline PCO (\%) & 57.8 & & 52.7 & & 64.8 & & 0.168 \\
\hline Clinical hyperandrogenism (\%) & 71.5 & & 71.5 & & 81.2 & & 0.242 \\
\hline $\begin{array}{l}\text { Biochemical } \\
\text { hyperandrogenism (\%) }\end{array}$ & 52.7 & & 48.3 & & 51.6 & & 0.617 \\
\hline
\end{tabular}

$* P<0.05$ for negative $\mathrm{FHx}$ vs positive FHx of T2DM or PCOS (Mann-Whitney $U$ test) ${ }^{\dagger} P<0.05$ for negative $\mathrm{FHx}$ vs positive $\mathrm{FHx}$ of T2DM and PCOS (MannWhitney $U$ test); ${ }^{*} P<0.05$ for positive FHx of T2DM or PCOS vs positive FHx of T2DM and PCOS (Mann-Whitney $U$ test). PCOS, polycystic ovary syndrome; FHx, family history; T2DM, type 2 diabetes mellitus; WC, waist circumference; WHR, waist-to-hip ratio; BP, blood pressure; AUC, area under the curve; HOMA, homeostatic model assessment; OUICKI, quantitative insulin sensitivity check index; ISI, insulin sensitivity index; LAP, lipid accumulation product; VAI, visceral adiposity index; SHBG, sex hormone-binding globulin; FAI, free androgen index; FG, Ferriman-Gallwey; TC, total cholesterol; TG, triglycerides; MS, metabolic syndrome; PCO, polycystic ovaries.

Binary logistic regression analyses using metabolic variables as dependent variables were attenuated (except prediabetes (OR for T2DM FHx: 2.26, 1.03-4.98; $P=0.040)$ ). In contrast, binary regression analysis remained stable for PCOS FHx and clinical hyperandrogenism (OR 4.11, 1.16-14.58; $P=0.019)$, hirsutism (OR 3.66, 1.31-10.20; $P=0.013)$, elevated FAI
(OR 2.95, 1.03-8.44; $P=0.044)$ and elevated FT (OR 2.32, 1.02-5.27; $P=0.044)$.

\section{Healthy women}

Baseline characteristics of the control group have been reported previously (24). The prevalence of T2DM FHx was 
significantly lower in control women $(16.2 \%, P<0.001)$ compared with PCOS women. We found no significant differences in metabolic or endocrine parameters between healthy women with and without T2DM FHx $(P>0.100$ for all, data not shown).
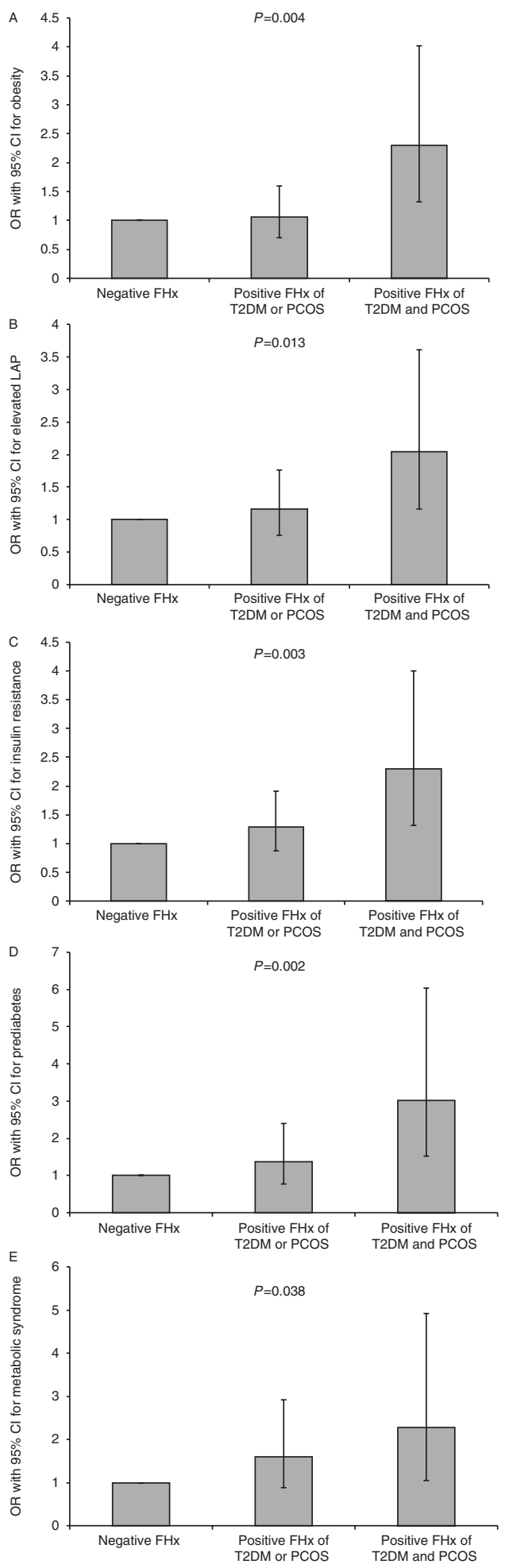

\section{Discussion}

We present evidence that a positive FHx of T2DM is independently associated with metabolic disturbances such as central fat accumulation, obesity, prediabetes, MS, insulin resistance dyslipidaemia and high BP in a large cohort of PCOS women. A positive FHx of PCOS, however, was independently associated with clinical and biochemical hyperandrogenism and prediabetes. Of note, PCOS women with a positive FHx of both T2DM and PCOS had the highest prevalence of metabolic disturbances and hyperandrogenism.

Our results indicate that FHx of T2DM and PCOS have a different influence on PCOS phenotype. FHx of T2DM was independently associated with obesity, central fat accumulation, MS, IR and prediabetes, dyslipidaemia and arterial hypertension. In contrast, PCOS FHx was only related to prediabetes but showed no significant independent association with other metabolic parameters. These findings might have several implications for clinical practice as well as for future research. First and clinically most important, the simple assessment whether a relative is affected by T2DM or not might allow risk stratification of PCOS women. It might help identify PCOS women at high metabolic risk in whom further evaluation including a regular follow-up as well as intensified treatment are indicated. Second, a positive FHx of T2DM might identify a distinct subgroup of PCOS women affected by central fat

\section{Figure 1}

OR with $95 \% \mathrm{Cl}$ for obesity, central fat accumulation, insulin resistance, prediabetes and MS in PCOS women stratified by positive FHx of T2DM and PCOS. Binary logistic regression analyses using all-factors models with obesity, prediabetes and MS as dependent variables adjusted for age and testosterone (negative $\mathrm{FHx}$ of both diseases was defined as reference). Negative FHx: $n=336$; positive FHx of T2DM or PCOS: $n=226$; positive FHx of T2DM and PCOS: $n=71$. OR, odds ratio; MS, metabolic syndrome; PCOS, polycystic ovary syndrome; $\mathrm{FHx}$, family history; T2DM, type 2 diabetes mellitus; LAP, lipid accumulation product. (A) OR with $95 \% \mathrm{Cl}$ for obesity depending on a positive FHx of T2DM and PCOS. (B) OR with 95\% Cl for elevated LAP depending on a positive FHx of T2DM and PCOS. (C) OR with $95 \% \mathrm{Cl}$ for insulin resistance depending on a positive FHx of T2DM and PCOS. (D) OR with $95 \% \mathrm{Cl}$ for prediabetes depending on a positive FHx of T2DM and PCOS. (E) OR with $95 \% \mathrm{Cl}$ for MS depending on a positive $\mathrm{FHx}$ of T2DM and PCOS. 
Table 6 Metabolic characteristics of non-obese and obese PCOS women. Analyses were carried out using $\chi^{2}$ test.

\begin{tabular}{|c|c|c|c|}
\hline & $\begin{array}{c}\text { Non-obese } \\
\text { PCOS women } \\
(n=533)\end{array}$ & $\begin{array}{c}\text { Obese PCOS } \\
\text { women }(n=181)\end{array}$ & $P$ value \\
\hline LAP elevated (\%) & 6.6 & 70.9 & $<0.001$ \\
\hline VAl elevated (\%) & 11.4 & 56.6 & $<0.001$ \\
\hline Prediabetes (\%) & 8.2 & 25.7 & $<0.001$ \\
\hline T2DM (\%) & 0.4 & 4.5 & $<0.001$ \\
\hline MS (\%) & 2.7 & 41.3 & $<0.001$ \\
\hline Insulin resistance (\%) & 9.7 & 60.0 & $<0.001$ \\
\hline TG elevated (\%) & 5.8 & 24.5 & $<0.001$ \\
\hline HDL low (\%) & 10.3 & 45.9 & $<0.001$ \\
\hline Elevated BP (\%) & 27.3 & 66.2 & $<0.001$ \\
\hline
\end{tabular}

PCOS, polycystic ovary syndrome; LAP, lipid accumulation product; VAI, visceral adiposity index; T2DM, type 2 diabetes mellitus; MS, metabolic syndrome; TG, triglycerides; $\mathrm{BP}$, blood pressure.

accumulation and metabolic disturbances. Those women differ from women without a positive FHx of T2DM, which might be related to either genetic or environmental factors. Of note, it has been shown that T2DM-related genetic variants are also associated with PCOS phenotype and risk $(3,15)$. To our knowledge, it has not been investigated so far whether PCOS women with a positive FHx of T2DM have a higher prevalence of T2DM-related genetic variants than PCOS women with a negative T2DM FHx. Thus, future genetic studies on PCOS phenotype should also analyse the association of positive T2DM FHx with those genetic variants. We found an about 1.7-fold increased risk of prediabetes and insulin resistance and twofold increased risk of MS and dyslipidaemia in PCOS women with a positive FHx of T2DM. Thus, our findings are consistent with a genetic basis for metabolic disturbances in PCOS, which has been suggested previously $(15,25,26)$. Interestingly, PCOS women with a positive T2DM FHx were more likely to have a positive PCOS FHx suggesting a common genetic background of both diseases. The hypothesis of a strong genetic background of PCOS and T2DM is supported by a study among 200 parents of PCOS women and 120 parents of healthy young women showing that parents of PCOS women are more insulin resistant and have a higher prevalence of T2DM (27). Similarly, a higher prevalence of T2DM and insulin resistance has been found in 102 first-degree relatives of PCOS women compared with 82 healthy controls without PCOS FHx (28).

Prediabetes and MS were prevalent in 16.9 and 16.4\% of women with a positive $\mathrm{FHx}$ of $\mathrm{T} 2 \mathrm{DM}$ respectively, whereas only 10.4 and $8.6 \%$ of PCOS women without FHx of T2DM presented with prediabetes and MS respectively. Legro et al. (12) observed a borderline significant difference in disturbed glucose metabolism that was prevalent in $52.6 \%$ of PCOS women with a FHx of T2DM and in 34\% of PCOS women with negative FHx (among 254 women with PCOS). The study was conducted in the US and the obesity prevalence was much higher (mean BMI $35.9 \mathrm{~kg} / \mathrm{m}^{2}$ ) than in the present study among Austrian PCOS women with a median of BMI $24.2 \mathrm{~kg} / \mathrm{m}^{2}$. Further, Ehrmann et al. (13) found a 2.6-fold higher prevalence of first-degree relatives with T2DM in 12 PCOS women with T2DM compared with 67 PCOS women with normal glucose tolerance (83 vs 31\%, $P<0.01$ ). That study again differed from our cohort with respect to BMI (mean BMI 33.4, 36.9 and $41.0 \mathrm{~kg} / \mathrm{m}^{2}$ for women with normal glucose tolerance, IGT and T2DM respectively) (13). A larger study including 408 women with PCOS found a positive FHx of T2DM in 44\% of the 16 diabetic PCOS women, 39\% of the 94 women with impaired glucose tolerance and $21 \%$ of the 298 women with normal glucose tolerance $(P<0.01)(14)$. Conversely, the prevalence of IGT and T2DM was significantly higher in PCOS women with a positive $\mathrm{FHx}$ of T2DM, which is in line with the findings of our study. Again, that study was conducted in the US and included women with a mean BMI of $36.2 \mathrm{~kg} / \mathrm{m}^{2}$ (14).

Interestingly, Ehrmann et al. (29) found that a positive FHx of T2DM in first-degree relatives defines a subset of PCOS women with a greater prevalence of insulin secretory defects that might be associated with an increased risk for developing T2DM in later life. Similarly, T2DM FHx has been shown to be associated with insulin resistance in 112 PCOS women from Spain (30) as well as in 37 Indian PCOS women (31). This notion is supported by our finding suggesting an independent association of T2DM FHx with insulin resistance in PCOS women.

Our results showing an increased metabolic risk of PCOS women with a positive FHx of both T2DM and PCOS are of high clinical impact. The prevalence of MS in that subgroup was $18 \%, 26 \%$ presented with prediabetes, $35 \%$ had central fat accumulation and $38 \%$ were obese. In clinical routine the simple question whether relatives are affected by T2DM and/or PCOS might be a useful screening tool to identify PCOS women at high metabolic risk. In our opinion, a 2-h OGTT should be carried out in all women with PCOS (32), which has also been suggested by the new Endocrine Society Clinical Practice Guideline (33). However, there might be situations when this is not possible and this time-consuming test is available only in a subgroup of PCOS women. Given the fact that PCOS has a 
very high prevalence, there is a need for simple and cheap screening tools to identify PCOS women at high metabolic risk. Those women should receive adequate diagnostic testing such as 2-h OGTT and intensive treatment such as lifestyle counselling and insulin-sensitising drugs. Of note, the recent Androgen Excess Society statement suggests that 2-h OGTT should be carried out in all PCOS women with a positive $\mathrm{FHx}$ of T2DM, recognising the importance of positive $\mathrm{FHx}$ as a risk factor for the development of disturbed glucose metabolism (23).

PCOS and the MS appear to be interrelated, although they are distinct entities. Women with PCOS are commonly affected by MS, while women with MS may display reproductive or endocrine features of PCOS. Thus, one might speculate on a mutual pathophysiologic relationship and potentially significant clinical sequelae. This relationship seems, however, to be largely mediated via obesity as only $2.7 \%$ of non-obese, but $41.3 \%$ of obese PCOS women in our study were affected by the MS. This is in accordance with relatively lean PCOS cohorts from Southern Europe showing a prevalence of MS between 8 and 25\% $(34,35)$. In contrast, the estimated prevalence of the MS in the USA is $33-47 \%$ and 13.7 times more likely in women with PCOS in the highest vs the lowest BMI $(23,36)$. This notion is supported by a study among 469 South Asian PCOS women with increasing MS prevalence from the lowest (6.7\%) to highest (42.0\%) quartiles of BMI and a relatively high overall prevalence of $36 \%$. Further, central obesity and visceral fat accumulation have been associated as key factors in the development of the MS, and the prevalence of the MS is low if there is no excessive abdominal adiposity $(8,9)$. Moreover, non-alcoholic fatty liver disease (NAFLD) is considered the hepatic manifestation of MS and it has been shown that $89 \%$ of PCOS women with MS have elevated fatty liver index indicating NAFLD (24). Besides obesity and insulin resistance, an inflammatory atherothrombotic state with elevated proinflammatory substances (such as C-reactive protein, fibrinogen, white blood cells, plasminogen activator inhibitor-1, and endothelin-1) has also been suggested in MS, which impair endothelial function, reduce vasoreactivity and promote subclinical atherosclerosis (23). Interestingly, it has also been demonstrated that genetic variants involved in atherothrombotic diseases and blood coagulation, such as the F13A1 gene, are associated with the metabolic phenotype in PCOS (37). Thus, a common genetic background of PCOS and the MS might be related to obesity as well as to other factors such as an atherothrombotic state.

We found no significant association of T2DM FHx with metabolic or endocrine parameters in control women. This might be related to the smaller sample size or to the fact that the prevalence of T2DM FHx was lower in healthy women (16.2 vs $36.8 \%)$. Further, the prevalence of metabolic disturbances is low in those healthy young women (24). Considering the lack of significance one might also speculate that the impact of T2DM FHx is larger in PCOS compared with BMI-matched women without hyperandrogenaemia.

Considering the higher prevalence of obesity in PCOS women with T2DM FHx, we tried to explore whether our findings are related to a genetic background or rather to lifestyle factors following a non-genetic model. The attenuation of some of our results in subgroup analyses of obese and non-obese women is in favour of a non-genetic model. This weakening might, however, also be related to the smaller sample size and the low prevalence of metabolic disturbances in non-obese women. Interestingly, most associations of PCOS FHx and metabolic and endocrine parameters remained stable in obese and nonobese women, suggesting that this relationship is mediated via genetic rather than lifestyle factors. Further, as some of our results remained significant and due to the fact that we found no significant association in healthy women, one should also consider the possibility of an important impact of FHx and therefore genetics in PCOS women. Further, the assumption of a common genetic background of PCOS and T2DM is strengthened by our results showing that both obese as well as non-obese PCOS women with a positive T2DM FHx are more likely to have a positive PCOS FHx.

Interestingly, a positive FHx of PCOS was closely related to biochemical and clinical hyperandrogenism in our PCOS cohort. In contrast, when analysing metabolic parameters, the only independent association was found with prediabetes. Thus, our findings suggest that women with a positive FHx of PCOS might constitute a distinct subgroup of PCOS women with an increased risk of hyperandrogenism but a relatively low risk of insulin resistance or MS. Thus, future genetic studies should also focus on the relationship of hyperandrogenism-related genotypes with PCOS FHx.

One limitation of our study is the fact that we assessed FHx of T2DM and PCOS via questionnaires, but we did not obtain information via medical records of family members. Further, relatives of PCOS women were not tested individually. Thus, we cannot exclude under- or over-reporting of PCOS or T2DM and one might consider a recall bias. As the diagnosis of PCOS might have been missed in mothers of PCOS women at their premenopausal age, it is likely that the prevalence of positive PCOS 
FHx is underestimated in the present study. However, as a clear definition of postmenopausal PCOS is missing, we are unable to make a definite diagnosis of PCOS in most mothers of the present PCOS population. Further, the use of patient recall rather than direct testing of family members is a common approach in studies assessing FHx and this is especially true for studies including a large sample size.

Nevertheless, it is possible that the use of questionnaires to assess FHx has a negative impact on the reliability of our findings. It is worth mentioning that our definition of a positive FHx of T2DM was not only restricted to firstdegree relatives but also included grandparents. Thus, our results might differ from other studies among PCOS cohorts that only included first-degree relatives. Further, the increased risk of metabolic disturbances might be even more pronounced when only first-degree relatives with a positive PCOS FHx are included. As PCOS was diagnosed according to the Rotterdam criteria, our findings might differ from other PCOS cohorts diagnosed by different criteria (AES or NIH) that might present with a more severe metabolic phenotype $(38,39)$. Of note, a more favourable metabolic profile has been suggested in nonhyperandrogenic PCOS women (23). Thus, our results are restricted to PCOS women fulfilling the Rotterdam criteria. Nevertheless, previous results from our PCOS cohort have demonstrated that the prevalence of metabolic disturbances including prediabetes and T2DM is similar in PCOS women with and without hyperandrogenism (32). Likewise, the prevalence of the MS and its components has been shown to be similar in PCOS women fulfilling Rotterdam criteria and AES criteria (1). Further, the Endocrine Society has recently suggested in their clinical practice guidelines to use the Rotterdam criteria for diagnosing PCOS (33). Another limitation is the fact that we have no information on PCOS FHx in control women. Moreover, we did not use the hyperinsulinaemic, euglycaemic glucose clamp technique, which is considered gold standard when assessing insulin resistance and sensitivity in vivo (40). It is, however, difficult to perform this time-consuming expensive clamp technique in large studies. Strengths of our study include the large sample size and the performance of an OGTT with measurements of glucose and insulin concentrations in all women. We were therefore able to calculate ISI (Matsuda) that correlates reasonably well with the estimates of whole-body insulin sensitivity determined by the glucose clamp and has recently been validated using glucose clamp studies (20).

In summary, we present evidence that PCOS women with a positive T2DM FHx have an adverse metabolic profile, whereas PCOS women with a positive PCOS FHx have increased prevalence of clinical and biochemical hyperandrogenism. PCOS women with a positive FHx of both T2DM and PCOS had the highest prevalence of metabolic and endocrine disturbances. Our findings suggest that the assessment of FHx might allow risk stratification of PCOS women, which is important considering the high prevalence of PCOS. Future studies on the genetic background of PCOS might focus on FHx of PCOS and T2DM.

\section{Declaration of interest}

The authors declare that there is no conflict of interest that could be perceived as prejudicing the impartiality of the research reported.

\section{Funding}

This research did not receive any specific grant from any funding agency in the public, commercial or not-for-profit sector.

\section{Author contribution statement}

E Lerchbaum contributed to study conception and design, acquisition and analysis of data, and drafting of article; V Schwetz contributed to acquisition of data and revising the article for important intellectual content; A Giuliani contributed to acquisition of data and revising the article for important intellectual content; B Obermayer-Pietsch contributed to conception, design, acquisition of data and drafting the article. All authors approved the final version of the manuscript.

\section{References}

1 Yildiz BO, Bozdag G, Yapici Z, Esinler I \& Yarali H. Prevalence, phenotype and cardiometabolic risk of polycystic ovary syndrome under different diagnostic criteria. Human Reproduction 201227 3067-3073. (doi:10.1093/humrep/des232)

2 Franks S \& McCarthy M. Genetics of ovarian disorders: polycystic ovary syndrome. Reviews in Endocrine and Metabolic Disorders 20045 69-76. (doi:10.1023/B:REMD.0000016125.05878.96)

3 Wojciechowski P, Lipowska A, Rys P, Ewens KG, Franks S, Tan S, Lerchbaum E, Vcelak J, Attaoua R, Straczkowski M et al. Impact of FTO genotypes on BMI and weight in polycystic ovary syndrome: a systematic review and meta-analysis. Diabetologia 201255 2636-2645. (doi:10.1007/s00125-012-2638-6)

4 Kahsar-Miller M \& Azziz R. The development of the polycystic ovary syndrome: family history as a risk factor. Trends in Endocrinology and Metabolism 19989 55-58. (doi:10.1016/S1043-2760(98)00021-6)

5 Escobar-Morreale HF, Luque-Ramírez M \& San Millán JL. The molecular-genetic basis of functional hyperandrogenism and the polycystic ovary syndrome. Endocrine Reviews 200526 251-282. (doi:10.1210/er.2004-0004)

6 Ehrmann DA. Polycystic ovary syndrome. New England Journal of Medicine 2005352 1223-1236. (doi:10.1056/NEJMra041536)

7 Wehr E, Möller R, Horejsi R, Giuliani A, Kopera D, Schweighofer N, Groselj-Strele A, Pieber TR \& Obermayer-Pietsch B. Subcutaneous adipose tissue topography and metabolic disturbances in polycystic ovary syndrome. Wiener Klinische Wochenschrift 2009121 262-269. (doi:10.1007/s00508-009-1162-2) 
8 Wehr E, Gruber HJ, Giuliani A, Möller R, Pieber TR \& ObermayerPietsch B. The lipid accumulation product is associated with impaired glucose tolerance in PCOS women. Journal of Clinical Endocrinology and Metabolism 201196 E986-E990. (doi:10.1210/jc.2011-0031)

9 Lerchbaum ESV, Giuliani A \& Obermayer-Pietsch B. Hypertriglyceridemic waist is associated with impaired glucose tolerance in polycystic ovary syndrome. Nutrition, Metabolism, and Cardiovascular Diseases 2013 23 e15-e16. (doi:10.1016/j.numecd.2012.11.004)

10 Wehr E, Pilz S, Boehm BO, März W \& Obermayer-Pietsch B. The lipid accumulation product is associated with increased mortality in normal weight postmenopausal women. Obesity 201119 1873-1880. (doi:10.1038/oby.2011.42)

11 Amato MC, Guarnotta V, Forti D, Donatelli M, Dolcimascolo S \& Giordano C. Metabolically healthy polycystic ovary syndrome (MH-PCOS) and metabolically unhealthy polycystic ovary syndrome (MU-PCOS): a comparative analysis of four simple methods useful for metabolic assessment. Human Reproduction 201328 1919-1928. (doi:10.1093/humrep/det105)

12 Legro RS, Kunselman AR, Dodson WC \& Dunaif A. Prevalence and predictors of risk for type 2 diabetes mellitus and impaired glucose tolerance in polycystic ovary syndrome: a prospective, controlled study in 254 affected women. Journal of Clinical Endocrinology and Metabolism 199984 165-169.

13 Ehrmann DA, Barnes RB, Rosenfield RL, Cavaghan MK \& Imperial J. Prevalence of impaired glucose tolerance and diabetes in women with polycystic ovary syndrome. Diabetes Care 199922 141-146. (doi:10.2337/diacare.22.1.141)

14 Ehrmann DA, Kasza K, Azziz R, Legro RS, Ghazzi MN \& PCOS/Troglitazone Study Group. Effects of race and family history of type 2 diabetes on metabolic status of women with polycystic ovary syndrome. Journal of Clinical Endocrinology and Metabolism 200590 66-71. (doi:10.1210/jc.2004-0229)

15 Wehr E, Schweighofer N, Möller R, Giuliani A, Pieber TR \& ObermayerPietsch B. Association of FTO gene with hyperandrogenemia and metabolic parameters in women with polycystic ovary syndrome. Metabolism 201059 575-580. (doi:10.1016/j.metabol.2009.08.023)

16 Graupp M, Wehr E, Schweighofer N, Pieber TR \& Obermayer-Pietsch B. Association of genetic variants in the two isoforms of $5 \alpha$-reductase, SRD5A1 and SRD5A2, in lean patients with polycystic ovary syndrome. European Journal of Obstetrics Gynecology and Reproductive Biology 2011 157 175-179. (doi:10.1016/j.ejogrb.2011.03.026)

17 The Rotterdam ESHRE/ASRM-Sponsored Consensus Workshop Group. Revised 2003 consensus on diagnostic criteria and long-term health risks related to polycystic ovary syndrome (PCOS). Human Reproduction 200419 41-47. (doi:10.1093/humrep/deh098)

18 Vermeulen A, Verdonck L \& Kaufman JM. A critical evaluation of simple methods for the estimation of free testosterone in serum. Journal of Clinical Endocrinology and Metabolism 199984 3666-3672. (doi:10.1210/jcem.84.10.6079)

19 Matsuda M \& DeFronzo RA. Insulin sensitivity indices obtained from oral glucose tolerance testing: comparison with the euglycemic insulin clamp. Diabetes Care 199922 1462-1470. (doi:10.2337/diacare.22.9. 1462)

20 Abdul-Ghani MA, Matsuda M, Balas B \& DeFronzo RA. Muscle and liver insulin resistance indexes derived from the oral glucose tolerance test. Diabetes Care 200730 89-94. (doi:10.2337/dc06-1519)

21 American Diabetes Association. Standards of medical care in diabetes 2013. Diabetes Care 201336 S11-S66. (doi:10.2337/dc13-S011)

22 Grundy SM, Cleeman JI, Daniels SR, Donato KA, Eckel RH, Franklin BA, Gordon DJ, Krauss RM, Savage PJ, Smith SC Jr et al. Diagnosis and management of the metabolic syndrome: an American Heart Association/National Heart, Lung, and Blood Institute Scientific Statement. Circulation 2005112 2735-2752. (doi:10.1161/CIRCULATIONAHA. 105.169404)

23 Wild RA, Carmina E, Diamanti-Kandarakis E, Dokras A, EscobarMorreale HF, Futterweit W, Lobo R, Norman RJ, Talbott E \&
Dumesic DA. Assessment of cardiovascular risk and prevention of cardiovascular disease in women with the polycystic ovary syndrome: a consensus statement by the Androgen Excess and Polycystic Ovary Syndrome (AE-PCOS) Society. Journal of Clinical Endocrinology and Metabolism 201095 2038-2049. (doi:10.1210/jc.2009-2724)

24 Lerchbaum E, Gruber HJ, Schwetz V, Giuliani A, Möller R, Pieber TR \& Obermayer-Pietsch B. Fatty liver index in polycystic ovary syndrome. European Journal of Endocrinology 2011165 935-943. (doi:10.1530/ EJE-11-0614)

25 Wehr E, Trummer O, Giuliani A, Gruber HJ, Pieber TR \& ObermayerPietsch B. Vitamin D-associated polymorphisms are related to insulin resistance and vitamin D deficiency in polycystic ovary syndrome. European Journal of Endocrinology 2011164 741-749. (doi:10.1530/ EJE-11-0134)

26 Lerchbaum E, Trummer O, Giuliani A, Gruber HJ, Pieber TR \& Obermayer-Pietsch B. Susceptibility loci for polycystic ovary syndrome on chromosome 2p16.3, 2p21, and 9q33.3 in a cohort of Caucasian women. Hormone and Metabolic Research 201143 743-747. (doi:10.1055/s-0031-1286279)

27 Sir-Petermann T, Angel B, Maliqueo M, Carvajal F, Santos JL \& PérezBravo F. Prevalence of type II diabetes mellitus and insulin resistance in parents of women with polycystic ovary syndrome. Diabetologia 2002 45 959-964. (doi:10.1007/s00125-002-REF30=10.1016/j.fertnstert. 2009.11.049)

28 Yildiz BO, Yarali H, Oguz H \& Bayraktar M. Glucose intolerance, insulin resistance, and hyperandrogenemia in first degree relatives of women with polycystic ovary syndrome. Journal of Clinical Endocrinology and Metabolism 200388 2031-2036. (doi:10.1210/jc.2002-021499)

29 Ehrmann DA, Sturis J, Byrne MM, Karrison T, Rosenfield RL \& Polonsky KS. Insulin secretory defects in polycystic ovary syndrome. Relationship to insulin sensitivity and family history of non-insulindependent diabetes mellitus. Journal of Clinical Investigation 199596 520-527. (doi:10.1172/JCI118064)

30 Luque-Ramírez M, Alpañés M \& Escobar-Morreale HF. The determinants of insulin sensitivity, $\beta$-cell function, and glucose tolerance are different in patients with polycystic ovary syndrome than in women who do not have hyperandrogenism. Fertility and Sterility 201094 2214-2221. (doi:10.1016/j.fertnstert.2REF34=10.1530/eje.1 02058)

31 Kalra P, Bansal B, Nag P, Singh JK, Gupta RK, Kumar S, Rathore RK, Bhatia V \& Bhatia E. Abdominal fat distribution and insulin resistance in Indian women with polycystic ovarian syndrome. Fertility and Sterility 200991 1437-1440. (doi:10.1016/j.fertnstert. 2008.06.037)

32 Lerchbaum E, Schwetz V, Giuliani A \& Obermayer-Pietsch B. Assessment of glucose metabolism in polycystic ovary syndrome: $\mathrm{HbA1c}$ and fasting glucose versus oral glucose tolerance test as screening method. Human Reproduction 201328 2537-2544. (doi:10.1093/humrep/det255)

33 Legro RS, Arslanian SA, Ehrmann DA, Hoeger KM, Murad MH, Pasquali R \& Welt CK. Diagnosis and treatment of polycystic ovary syndrome: an Endocrine Society clinical practice guideline. Journal of Clinical Endocrinology and Metabolism 201398 4565-4592. (doi:10.1210/ jc.2013-2350)

34 Carmina E, Napoli N, Longo RA, Rini GB \& Lobo RA. Metabolic syndrome in polycystic ovary syndrome (PCOS): lower prevalence in southern Italy than in the USA and the influence of criteria for the diagnosis of PCOS. European Journal of Endocrinology 2006154 141-145. (doi:10.1530/eje.1.02058)

35 Alvarez-Blasco F, Botella-Carretero JI, San Millán JL \& EscobarMorreale HF. Prevalence and characteristics of the polycystic ovary syndrome in overweight and obese women. Archives of Internal Medicine 2006166 2081-2086. (doi:10.1001/archinte.166.19.2081)

36 Ehrmann DA, Liljenquist DR, Kasza K, Azziz R, Legro RS, Ghazzi MN \& PCOS/Troglitazone Study Group. Prevalence and predictors of the metabolic syndrome in women with polycystic ovary syndrome. 
Journal of Clinical Endocrinology and Metabolism 200691 48-53. (doi:10.1210/jc.2005-1329)

37 Schweighofer N, Lerchbaum E, Trummer O, Schwetz V, Pilz S, Pieber TR \& Obermayer-Pietsch B. Androgen levels and metabolic parameters are associated with a genetic variant of F13A1 in women with polycystic ovary syndrome. Gene 2012504 133-139. (doi:10.1016/j.gene.2012.04.050)

38 Carmina E, Chu MC, Longo RA, Rini GB \& Lobo RA. Phenotypic variation in hyperandrogenic women influences the findings of abnormal metabolic and cardiovascular risk parameters. Journal of
Clinical Endocrinology and Metabolism 200590 2545-2549. (doi:10.1210/ jc.2004-2279)

39 Livadas S \& Diamanti-Kandarakis E. Polycystic ovary syndrome: definitions, phenotypes and diagnostic approach. Frontiers of Hormone Research 201340 1-21.

40 Diamanti-Kandarakis E \& Dunaif A. Insulin resistance and the polycystic ovary syndrome revisited: an update on mechanisms and implications. Endocrine Reviews 201233 981-1030. (doi:10.1210/er. 2011-1034)

Received 17 December 2013

Revised version received 24 February 2014

Accepted 3 March 2014 